\title{
Meaning is in the beholder's eye: Morpho-semantic effects in masked priming
}

\author{
Marco Marelli • Simona Amenta • \\ Elena Angela Morone • Davide Crepaldi
}

Published online: 19 December 2012

(C) Psychonomic Society, Inc. 2012

\begin{abstract}
A substantial body of literature indicates that, at least at some level of processing, complex words are broken down into their morphemes solely on the basis of their orthographic form (e.g., Rastle, Davis, \& New, Psychonomic Bulletin and Review 11:1090-1098, 2004). Recent evidence has shown that this process might not be obligatory, as indicated by the fact that morpho-orthographic effects were not found in a cross-case same-different task - that is, when lexical access was not necessarily required (Duñabeitia, Kinoshita, Carreiras, \& Norris, Language and Cognitive Processes 26:509-529, 2011). In this study, we employed a task that required understanding a series of words and, thus, implied lexical access. Masked primes were shown very briefly right before the appearance of the target word; prime-target pairs entertained a morphosemantic (dealer-DEAL), a morpho-orthographic (corner$C O R N$ ), or a purely orthographic (brothel-BROTH) relationship. Eye fixation times clearly indicated facilitation for transparent pairs, but not for opaque pairs (or for orthographic pairs, which were used as a baseline). Conversely, the usual morpho-orthographic pattern was found in a control experiment, employing a lexical decision task. These results indicate that the access to a morpho-orthographic level of representation is not always necessary for lexical identification, which challenges models of visual word identification that cannot account for task-induced effects.
\end{abstract}

Keywords Masked priming · Task effects . Morpho-orthographic segmentation $\cdot$ Eye tracking

M. Marelli · S. Amenta • E. A. Morone - D. Crepaldi Department of Psychology, University of Milano-Bicocca, Milan, Italy

M. Marelli $(\square)$

Center for Mind/Brain Sciences, University of Trento,

Corso Bettini 31, 38068 Rovereto, TN, Italy

e-mail: marco.marelli@unitn.it
There is wide agreement that morphologically complex words (like bakery, incorrect, or misunderstanding) are decomposed into their morphemic constituents during visual word recognition. However, there is no mutual consent as to how exactly segmentation unfolds. In this respect, one issue that has ignited a strong debate over the last few years concerns whether segmentation is influenced solely by orthographic factors or also by semantic information.

All studies adopting paradigms that tap into late processing stages - for example, long stimulus-onset asynchrony or cross-modal priming - indicate that semantics plays a crucial role in morphological decomposition, as evidenced by processing facilitation only for genuinely related prime-target pairs (punishment-PUNISH) as opposed to pseudo-related pairs (inventory-INVENT) (e.g., Marslen-Wilson, Tyler, Waksler, \& Older, 1994; Rastle, Davis, Marslen-Wilson, \& Tyler, 2000; Rueckl \& Aicher, 2008). These results indicate a level of morphological analysis that is sensitive to semantic transparency. However, because the above paradigms tap into late processing levels, these data are not incompatible with an early stage of morphological processing where semantic transparency does not play any role.

Along these lines, data from masked-priming experiments suggest the existence of a morpho-orthographic routine that parses letter strings into morphemes solely on the basis of their orthographic form. In two seminal studies, Longtin, Segui, and Hallé (2003) and Rastle, Davis, and New (2004) found significant priming effects when the relationship between prime and target was both semantically transparent (e.g., dealer-DEAL) and opaque (e.g., corner-CORN), but not in an orthographic control condition (e.g., brothel-BROTH). Although this pattern of results was confirmed in several other experiments (see Rastle \& Davis, 2008), it is still unclear whether priming is of the same magnitude in transparent and opaque pairs (Diependaele, Duñabeitia, Morris, \& Keuleers, 2011; Feldman, O’Connor, \& Moscoso del Prado Martin, 2009; Järvikivi, \& Pyykkönen, 2011). Critically, however, it 
is undisputed that opaque pairs yield more facilitation than orthographic pairs do in lexical decision masked-priming experiments, which is the critical comparison for proving the existence of a morpho-orthographic segmentation routine. Because this process is supposed to be in action before word identification takes place (i.e., at a prelexical level), it was always considered to be an obligatory step in the analysis of (pseudo-) complex letter strings.

The obligatoriness of morphological segmentation has been recently questioned by Duñabeitia et al. (2011). These authors employed a cross-case same-different task, which requires participants to judge whether a lowercase and an uppercase letter string, presented one after the other, are the same. By presenting the prime word for a very brief time between the reference word and the target, the authors found no morpho-orthographic (or morpho-semantic) effect; that is, equivalent facilitation emerged for dealer-DEAL, cor$n e r-C O R N$, and brothel-BROTH. Although this study has the important merit of showing that morpho-orthographic effects are sensitive to task manipulations even in maskedpriming paradigms, the cross-case same-different task used by Duñabeitia et al. does not necessarily require lexical access (one can easily judge whether two nonwords are identical, for example), and so these data are hardly informative as to how morpho-orthographic segmentation serves word identification. In other words, Duñabeitia et al. showed that morpho-orthographic processing is not necessarily in action any time a letter string is presented to the visual identification system; but this process might still be necessary in order to achieve lexical identification (see Crepaldi, Rastle, Coltheart, \& Nickels, 2010).

In order to address this issue, we devised a new task, adapted from Baayen and Marelli (2010). In this task, participants are asked to read and understand a word and a number presented simultaneously at the two lateral extremities of a computer screen. No action is required while participants are processing the stimuli; comprehension is tested off-line (i.e., after the stimuli have disappeared) on a proportion of trials through yes/no questions. The fixation point is located where the word will appear, so as to ensure that people will look at the word first. The stimuli are presented for a very brief time (700 ms) so that participants will be forced to move away their eyes from the word as quickly as possible in order to process the number before it disappears. By doing so, we make (reasonably) sure that the fixation time on the word is the shortest possible time necessary to gain all the relevant information from the stimulus; this measure is thus taken as an index of processing speed and is used as the dependent variable in our task. Crucially, a masked-priming paradigm has to be introduced in order to be sure to tap into those (early) levels associated to the morpho-orthographic segmentation. The priming manipulation applies as in the standard lexical decision paradigm: A forward mask is presented after the fixation point at the same location on the screen, immediately followed by the prime, which in turn is immediately followed by the target. Priming is measured as the difference between the fixation time on the target word when this is preceded by a related word and the fixation time on the target word when this is preceded by a matched, unrelated word.

An advantage of this procedure is that we measure an implicit index of word-processing time (fixation duration). This makes sure that the measure adopted is associated to word processing per se, excluding processing time related to decision making. Moreover, because this task requires the reader to understand the critical word, it clearly implies lexical access.

In order to guarantee a direct comparison with previous masked-priming studies, we applied to this task the same manipulation as that used in those studies (e.g., Longtin et al., 2003); that is, we compared priming in semantically transparent morphological pairs (e.g., dealer-DEAL), semantically opaque morphological pairs (e.g., corner-CORN), and purely orthographic pairs (e.g., brothel-BROTH). If morphoorthographic segmentation is obligatory for lexical access in reading toward understanding, we should observe the usual pattern according to which dealer-DEAL and corner-CORN yield more facilitation, as compared with brother-BROTH.

\section{Experiment 1}

\section{Method}

\section{Participants}

Twenty-seven students from the University of MilanoBicocca were recruited to participate in the study, in exchange for either credit courses or 2 Euros. Participants were all skilled readers and native speakers of Italian. They had normal or corrected-to-normal vision and no history of learning disability or neurological impairment.

\section{Apparatus}

An EyeLink 1000 eyetracker manufactured by SR Research Ltd. (Canada) was employed in order to monitor participants' eye movements during the experiment. A chinrest support was used to maintain the position of the head constant, while a desktop camera sampled the pupil position at a frequency of $1000 \mathrm{~Hz}$. The recording was monocular.

\section{Materials}

One hundred fifty prime-target pairs were selected from the Italian database CoLFIS (Bertinetto et al., 2005), equally assigned to three conditions. In the transparent condition, primes and targets entertained a genuine morphological 
relationship (e.g., artista-ARTE, artist-ART). In the opaque condition, primes and targets were semantically unrelated but entertained an apparent morphological relationship; in fact, primes were fully parsable in a leftmost portion (homograph to an existent root), which was shared with the target, and a rightmost portion (homograph to an existent suffix) (e.g., retaggio-RETE, legacy-NET; an analogous example in English would be corner-CORN). In the form condition, primes and targets had a purely orthographic relationship; that is, the stem of the target was homograph to the leftmost portion of the prime, whose ending did not correspond to a suffix (e.g., corallo-CORO, coral-CHOIR; an analogous example in English would be dialog-DIAL). One hundred fifty control primes were also chosen. These were existent, morphologically complex Italian words, which did not entertain any relationship (semantic, morphological, or visual) with the corresponding targets. Targets were matched as closely as possible across conditions for frequency, length, and orthographic neighborhood size $(N)$ (see Table 1a). Related primes and paired control primes were matched as closely as possible for the same variables (Table 1b). Moreover, we also matched across conditions the orthographic overlap between targets and the corresponding related and unrelated primes, calculated using the spatial coding for letter position (Davis, 2010; see Table 1b). Finally, we matched related primes for their letter transitional probabilities at the morphemic boundary (Table 1b).

The assignment of word targets to the two priming conditions was counterbalanced over participants, so that all participants received both related and control primes but saw each target only once. This was achieved by creating two experimental lists, which were submitted to different groups of participants.

\section{Procedure}

Trials began with a fixation point located in the upper-left quarter of a computer screen, in correspondence to the center of the following prime stimulus. A hash mark mask followed and remained on the screen for $500 \mathrm{~ms}$; this extended to the whole screen width, interrupted only by a cross superimposed on the fixation point. The mask was then substituted by the prime stimulus, which was presented in lowercase and remained on the screen for $35 \mathrm{~ms}$, along with a " 0 " in the right-hand portion of the screen. The exact position of the prime word was determined dynamically on each trial so that it was centered on the fixation point. Finally, a screen with the target stimulus was presented for $700 \mathrm{~ms}$. The uppercase target stimulus was presented in the same position as the prime, while a one-digit number (ranging from 1 to 8 ) was presented in the right-hand portion of the screen, symmetrical to the screen center. The appearance of the target screen triggered the registration of eye movements. The trial time line is represented in Fig. 1.
Table 1 Matching of relevant variables for targets and primes

\begin{tabular}{lllll}
\hline & & Condition & & \\
\cline { 3 - 5 } & & Transparent & Opaque & Form \\
\hline (a) Targets & & & & \\
Log-frequency & & $1.87 \pm 0.56$ & $1.45 \pm 0.82$ & $1.46 \pm 0.79$ \\
Length & & $5.44 \pm 1.21$ & $4.71 \pm 0.81$ & $5.12 \pm 1.02$ \\
Orthographic & & $5.22 \pm 3.56$ & $6.96 \pm 4.71$ & $7.58 \pm 4.31$ \\
neighborhood & & & & \\
size & & & & \\
(b) Primes & & & & \\
Log-frequency & (related) & $0.96 \pm 0.73$ & $1.04 \pm 0.74$ & $1.03 \pm 0.78$ \\
& (control) & $0.99 \pm 0.67$ & $1.08 \pm 0.67$ & $1.11 \pm 0.64$ \\
Length & (related) & $8.11 \pm 1.36$ & $8.18 \pm 1.45$ & $7.42 \pm 1.39$ \\
& (control) & $8.11 \pm 1.36$ & $8.16 \pm 1.47$ & $7.46 \pm 1.41$ \\
Orthographic & (related) & $0.92 \pm 1.01$ & $0.91 \pm 1.15$ & $1.24 \pm 1.44$ \\
neighborhood & (control) & $1.48 \pm 1.27$ & $1.31 \pm 1.52$ & $1.76 \pm 1.77$ \\
size & & & & \\
Orthographic & (related) & $0.81 \pm 0.11$ & $0.82 \pm 0.11$ & $0.80 \pm 0.12$ \\
$\quad$ overlap & (control) & $0.21 \pm 0.11$ & $0.21 \pm 0.12$ & $0.18 \pm 0.09$ \\
Bigram trough & (related) & $0.65 \pm 0.46$ & $0.65 \pm 0.41$ & $0.85 \pm 0.51$ \\
depth & & & & \\
\hline
\end{tabular}

Note. Frequency values refer to the raw number of occurrences in a corpus of 3,800,000 word forms (Bertinetto et al., 2005). Bigram trough depth was operationalized as $\left|\log B F_{a}-\log B F_{b}\right|+\left|\log B F_{c}-\log B F_{b}\right|$, where $B F_{a}$ is the frequency of the bigram immediately preceding the boundary, $B F_{b}$ is the frequency of the bigram straddling the boundary, and $B F_{c}$ is the frequency of the bigram immediatly after the boundary. Orthographic overlap was calculated using the spatial coding for letter position (Davis, 2010)

All elements were presented in gray Courier New case (32 point) on a black screen. For a mean viewing distance of $60 \mathrm{~cm}$, each character subtended $1.33^{\circ}$ of visual angle. About $15 \%$ of the trials were followed by a question regarding either the meaning of the word (e.g., does the word refer to a tool?) or the number (e.g., is the number odd?).

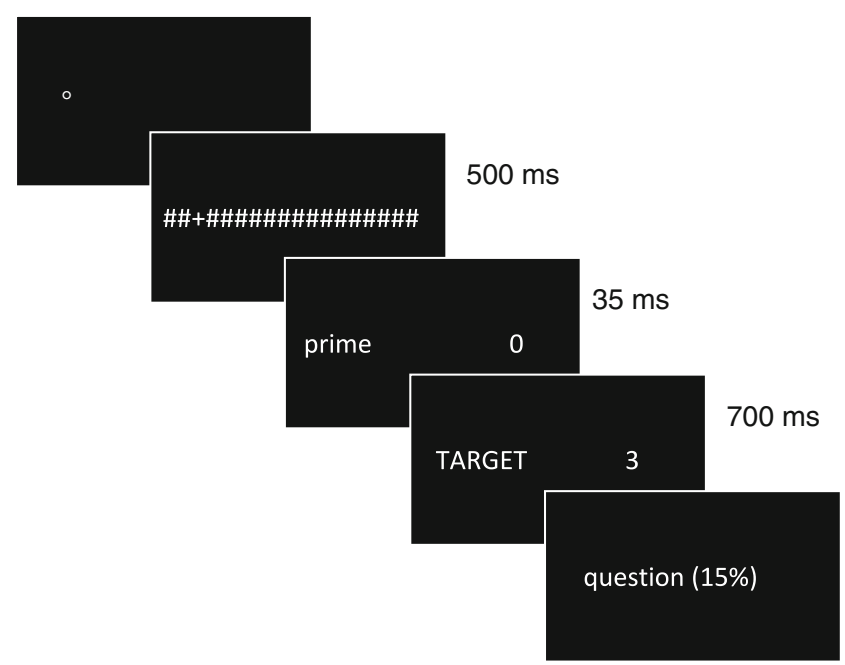

Fig. 1 Time line of trial presentation 
Participants were asked to read the word for comprehension and then look at the number. They were told that questions could be asked about either the word or the number at the end of each trial, and were instructed to answer them by pressing either a yes or a no button on a response pad. No mention was made of the presence of the prime words.

Before the experiment, the eyetracker was calibrated employing a three-point grid at the center of the screen. Before each trial, the fixation point was exploited to check fixation drift. In order to let the participants become familiar with the task, a practice session consisting of 10 trials was run at the beginning of the experiment. The whole experimental session lasted between 10 and $15 \mathrm{~min}$.

\section{Data analysis}

The durations of the first fixation and the gaze on the target word were adopted as dependent measures; gaze duration was defined as the total amount of time spent with the eyes on the stimulus, before fixating away from it. Mixed-effects models (Baayen, Davidson, \& Bates, 2008) were employed as the primary statistical tool. The effects of interest were those associated to the experimental manipulations - that is, relatedness (control vs. related prime), condition (transparent vs. opaque vs. form), and their mutual interaction. In order to account for more error variance, a number of covariates were also considered. Length, log-transformed frequency, and $N$ of both the prime and target, as well as trial number, were introduced in the initial model. Random intercepts for participants and items were also introduced. Effects were evaluated one by one on the basis of likelihood ratio tests; those whose inclusion did not increase significantly the model goodness of fit were removed from the analysis. After having identified the best model with this procedure, atypical outliers were identified and removed (employing 2.5 SDs of the residual errors as a criterion). Statistics in the refitted models are reported. The statistical significance of the fixed parameters was evaluated using Markov chain Monte Carlo sampling (pMCMC).

Results

Only data for fixations on target stimuli were considered. Fixations that either preceded or followed a blink were excluded from the analyses, as well as refixations on the targets (i.e., fixations that went back to the word after having fixated away from it, observed on $13 \%$ of the trials). Data points that deviated from a normal distribution were also excluded. The analyzed data set consisted of 3,905 valid data points.

The average number of fixations on the target was 1.6 $(S D=0.6)$, the average first-fixation duration was $256 \mathrm{~ms}$ $(S D=71)$, and the average gaze duration was $330 \mathrm{~ms}(S D=$ 88). Fifty percent of the targets required a single fixation for reading, $45 \%$ required two fixations, and $5 \%$ required either three or four fixations.

\section{First-fixation durations}

Table 2 summarizes the first-fixation durations in the different experimental situations. The interaction between relatedness and condition was significant, $F=4.26, p=.0142$. No significant priming effect emerged either in the form condition or in the opaque condition. However, a significant effect was found in the transparent condition: First-fixation durations were shorter for target words preceded by a morphosemantically related prime, in comparison with target words preceded by a control prime. Table 3 reports the parameters of the significant effects included in the final model.

\section{Gaze durations}

Table 4 summarizes the gaze durations in the different experimental situations. The interaction between relatedness and condition was significant, $F=6.25, p=.0019$. No significant priming effect emerged either in the form condition or in the opaque condition. However, a significant effect was found in the transparent condition: Gaze durations were shorter for target words preceded by a morphosemantically related prime, in comparison with target words preceded by a control prime. Table 5 reports the parameters of the significant effects included in the final model.

\section{Discussion}

The present results ideally complement the evidence provided by Duñabeitia et al. (2011) in showing that morphoorthographic effects are sensitive to task requirements. These authors employed a cross-case same-different task, which arguably taps into early orthographic processing and does not necessarily involve lexical access. Therefore, one may argue that morpho-orthographic effects could not be found in Duñabeitia et al.'s experiment because the processing stage at which morpho-orthographic segmentation occurs was not even reached. On the contrary, our experimental conditions entail semantic processing, which obviously requires lexical access. Thus, the lack of priming among opaque pairs in our

Table 2 Mean first-fixation durations and standard errors of the means (in milliseconds) in the different experimental situations

\begin{tabular}{|c|c|c|c|c|c|c|}
\hline & \multicolumn{2}{|c|}{ Transparent } & \multicolumn{2}{|c|}{ Opaque } & \multicolumn{2}{|c|}{ Form } \\
\hline & $M$ & SEM & $M$ & SEM & $M$ & SEM \\
\hline Control prime & 261 & 1.18 & 253 & 1.11 & 255 & 1.14 \\
\hline Related prime & 251 & 1.03 & 258 & 1.16 & 254 & 1.19 \\
\hline
\end{tabular}


Table 3 Fixed effects in the final model on first-fixation durations

\begin{tabular}{lrrrr}
\hline Fixed effect & Estimate & \multicolumn{1}{l}{$S E$} & $t$ value & pMCMC \\
\hline Intercept & 259.86 & 9.93 & 26.18 & .0001 \\
Relatedness: related & 0.55 & 3.18 & 0.17 & .8668 \\
Condition: opaque & 1.37 & 3.51 & 0.39 & .6984 \\
$\quad$ Condition: transparent & 9.29 & 3.56 & 2.61 & .0074 \\
$\quad \begin{array}{l}\text { Relatedness: related * } \\
\quad \text { condition: opaque }\end{array}$ & 2.66 & 4.44 & 0.61 & .5514 \\
$\quad$ Relatedness: related * & -9.61 & 4.44 & 2.16 & .0321 \\
$\quad$ condition: transparent & & & & \\
$\quad$ Target length & 3.76 & 1.46 & 2.58 & .0086 \\
$\quad$ Trial number & .05 & .02 & 2.38 & .0208 \\
\hline
\end{tabular}

Note. The reported covariates had significant effects and significantly improved the model goodness of fit.

experiment indicates not only that morpho-orthographic segmentation is not necessarily in place every time we are exposed to printed complex words, but also that this process is not always needed for lexical access.

Before drawing this conclusion, however, we need to confirm that the pattern of results that emerged in Experiment 1 would turn into the typical difference between morpho-orthographic and form conditions in a classical lexical decision masked-priming experiment. This would be direct evidence that this lack of morpho-orthographic effect does not depend on the specific items that we used, or perhaps on some peculiar feature of the Italian language (in fact, pseudo-derived words were never shown to prime their pseudo-stems in this language). To this aim, we ran a traditional masked-priming lexical decision experiment to confirm that there is some corn in the corner also in Italian.

\section{Experiment 2}

Method

\section{Participants}

Fifty-eight participants from the same population that took part in Experiment 1 were recruited to participate in the study. None of them had also taken part in Experiment 1.

Table 4 Mean gaze durations and standard errors of the means (in milliseconds) in the different experimental situations

\begin{tabular}{|c|c|c|c|c|c|c|}
\hline & \multicolumn{2}{|c|}{ Transparent } & \multicolumn{2}{|c|}{ Opaque } & \multicolumn{2}{|c|}{ Form } \\
\hline & $M$ & SEM & $M$ & SEM & $M$ & $S E M$ \\
\hline Control prime & 345 & 1.45 & 329 & 1.36 & 325 & 1.41 \\
\hline Related prime & 329 & 1.36 & 330 & 1.36 & 324 & 1.48 \\
\hline
\end{tabular}

Table 5 Fixed effects in the final model on gaze durations

\begin{tabular}{lrrrr}
\hline Fixed effect & Estimate & \multicolumn{1}{l}{$S E$} & $t$ value & pMCMC \\
\hline Intercept & 263.14 & 13.99 & 18.81 & .0001 \\
Relatedness: related & 1.16 & 3.34 & 0.35 & .7328 \\
Condition: opaque & -3.12 & 3.74 & 0.83 & .4028 \\
Condition: transparent & 5.89 & 3.88 & 1.52 & .1202 \\
Relatedness: related $*$ & -3.82 & 4.66 & 0.82 & .4224 \\
$\quad$ condition: opaque & & & & \\
Relatedness: related $*$ & -15.79 & 4.66 & 3.38 & .0006 \\
$\quad$ condition: transparent & & & & \\
Prime length & 3.51 & 1.21 & 2.89 & .0034 \\
Target length & 11.28 & 1.79 & 6.31 & .0001 \\
Target $N$ & -.98 & .36 & 2.72 & .0048 \\
Target frequency & -3.48 & 1.72 & 2.03 & .0384 \\
Trial number & -.08 & .02 & 3.72 & .0002 \\
\hline
\end{tabular}

Note. The reported covariates had significant effects and significantly improved the model goodness of fit

\section{Materials}

The same 150 prime-target pairs that were used in Experiment 1 were also used in this experiment. They were also counterbalanced over participants as in Experiment 1.

\section{Procedure}

Participants were seated in front of a computer screen and were instructed to decide whether the letter strings appearing on the screen were existing Italian words. They were informed that the target word would be preceded by a string of hash marks as a warning signal, but no mention was made about the presence of the prime word. In order to familiarize them with the task, participants were given six practice trials. Moreover, each experimental session began with five warm-up trials that were not analyzed so as to avoid outlier response due to unfamiliarity with the task.

Each trial started with a string of hash marks displayed for $500 \mathrm{~ms}$, which was followed by the prime word presented in lowercase for $35 \mathrm{~ms}$. The target word appeared on the screen immediately after the prime offset and remained on the screen until participants' response.

Stimulus presentation and data recording was accomplished via MATLAB and its Psychtoolbox functions. A response box was used to collect lexical decision times; the yes response button was always controlled by the participant's dominant hand.

\section{Data analysis}

Data were analyzed as in Experiment 1, with the only exception that response times were negative inverse transformed in order to make their distribution more Gaussian-like. 


\section{Results}

Table 6 reports mean response times in the experimental conditions. Mixed-effects models revealed an interaction between relatedness and condition, $F=31.79, p=.0001$ : Priming emerged in the opaque condition and in the transparent condition, but not in the orthographic condition (Table 7). Priming was also larger in the transparent than in the opaque condition (estimate $=-.06, \mathrm{pMCMC}=.0001)$.

\section{Discussion}

Results confirm that the same prime-target pairs as those used in Experiment 1 give rise to the traditional morphoorthographic effect in a standard lexical decision, maskedpriming study. Pseudo-derived words (e.g., brother) prime their pseudo-stems (e.g., broth) more than orthographic primes (e.g., brothel) do, thus proving that they are parsed into their morphemes on the basis of their orthographic form. Critically, this excludes the possibility that the lack of a morpho-orthographic effect in Experiment 1 was due to some peculiar features of Italian or of these specific items. Moreover, these results confirm that the traditional pattern of morpho-orthographic priming is observed also in a language that, differently from English, does not present free stems in derived words.

\section{General discussion}

The experiments described in this article show that it is possible to set task requirements so that morpho-orthographic effects do not emerge in a masked-priming environment - that is, where they are reliably reported in classical lexical decision tasks. Critically, and contrary to Duñabeitia et al. (2011), this was achieved with a task that requires lexical access, thus showing that morpho-orthographic segmentation is not always necessary for the visual identification of complex words. This conclusion challenges substantially existing models of visual word processing.

It is not clear which factor might have exactly determined the lack of morpho-orthographic effects in Experiment 1. In fact, as compared with the standard lexical decision

Table 6 Mean response times and standard errors of the means (in milliseconds) in the different experimental situations

\begin{tabular}{|c|c|c|c|c|c|c|}
\hline & \multicolumn{2}{|c|}{ Transparent } & \multicolumn{2}{|c|}{ Opaque } & \multicolumn{2}{|c|}{ Form } \\
\hline & $M$ & SEM & $M$ & SEM & $M$ & SEM \\
\hline Control prime & 613 & 1.63 & 637 & 1.69 & 642 & 1.81 \\
\hline Related prime & 575 & 1.49 & 620 & 1.82 & 641 & 1.93 \\
\hline
\end{tabular}

Table 7 Fixed effects in the final model on response times

\begin{tabular}{|c|c|c|c|c|}
\hline Fixed effect & Estimate & $S E$ & $t$ value & $\mathrm{pMCMC}$ \\
\hline Intercept & -1.48 & .03 & 46.52 & .0001 \\
\hline Relatedness: related & -0.02 & .01 & 1.68 & .0956 \\
\hline Condition: opaque & -0.01 & .02 & 0.63 & .5038 \\
\hline Condition: transparent & -0.04 & .02 & 2.11 & .0304 \\
\hline $\begin{array}{l}\text { Relatedness: related * } \\
\text { condition: opaque }\end{array}$ & -0.05 & .01 & 3.47 & .0002 \\
\hline $\begin{array}{l}\text { Relatedness: related } * \\
\text { condition: transparent }\end{array}$ & -0.11 & .01 & 7.94 & .0001 \\
\hline Target $N$ & -.01 & .01 & 1.88 & .0502 \\
\hline Target frequency & -.08 & .01 & 8.24 & .0001 \\
\hline
\end{tabular}

Note. The reported covariates had significant effects and significantly improved the model goodness of fit

experiment described in Experiment 2 (which led to the usual morpho-orthographic pattern), the Experiment 1 procedure employed (1) a new dependent variable (eye fixation time, rather than decision time) and (2) a new task (comprehension, rather than lexical decision). The latter factor is indeed more likely to be responsible for our data, since several experiments have documented task-induced changes of established patterns of results in masked priming (e.g., Bueno \& FrenckMestre, 2008; Norris \& Kinoshita, 2008). However, the crucial thing to note is that, independently of whether we blame the task or the dependent variable for the lack of morphoorthographic effects, the main point illustrated by this experiment remains valid: Morpho-orthographic segmentation is not always necessary to achieve lexical identification, because if it were, we would observe morpho-orthographic effects any time readers identify words - that is, in any task involving word identification and measuring any index of word identification time.

Clearly, the present results do not speak against the reliability of morpho-orthographic effects in lexical decision; those data are solid and surely indicate the existence of a processing level where morphemes are primarily identified on orthographic grounds. However, our data shed new light on the interpretation of this phenomenon. For example, most oneroute, localist models of the visual identification of complex words include a morpho-orthographic segmentation stage as an obligatory step in order to access the lexicon (Crepaldi et al., 2010; Taft, 2004), which is clearly not tenable given the present results. These models should be extended to account for the fact that morpho-orthographic segmentation is triggered by a lexical decision task but may be by-passed when one is trying to understand the meaning of a word (at least under the specific conditions imposed by our task).

Prima facie, models positing multiple processing routes (e.g., Grainger \& Ziegler, 2011; Kuperman, Schreuder, Bertram, \& Baayen, 2009) could account more easily for the present data, under the assumption that task requirements can 
modulate the relative importance of their different routes. However, the relationship between task requirements and processing strategies can be very intricate and is often underspecificied in these models. For example, although Kuperman et al. predicted that both form-based and semantic-based cues can be activated in parallel even before the whole word is read (see Marelli \& Luzzatti, 2012), it is not clear how task settings might affect these dynamics. Similarly, with reference to the model by Grainger and Ziegler, it is not clear why a morpheme-based route should operate when processing is focused mainly on lexicality and shut down when processing is focused mainly on comprehension.

Alternatively, a different perspective could be adopted in the way models are conceived. In place of fixed modules and rigid architectures, we could hypothesize a processing style characterized by temporary representations, employed ad hoc in relation to task requirements. For example, the present data might be explained in the context of the model for masked priming proposed by Norris and Kinoshita (2008) following the Bayesian Reader (Norris, 2006). In that framework, readers develop a series of hypotheses to keep under scrutiny in order to fulfill the requested tasks. These hypotheses are tested by processing the stimuli (both primes and targets) as evidence sources in accordance with the experimental conditions. Under these assumptions, it is reasonable to think that when people are to make lexical decisions, processing is focused on lexical and orthographic properties, with the semantic relationship between primes and targets being irrelevant. In these conditions, readers need to be quick and efficient in identifying words, and chunking frequent letter combinations-as (pseudo-)morphemes are-is an efficient strategy in this respect, no matter whether those chunks contribute to meaning (i.e., they are genuine morphemes) or not (i.e., they are pseudomorphemes). Conversely, when the task implies semantic access, processing would be focused on word meanings, and thus the semantic relationship between primes and targets would be crucial, irrespective of the morphological properties of the two words: Dealer will provide evidence for the meaning of DEAL, and the same will not happen for corner and CORN. Clearly, several details are lacking in this account, but its main principle fits well with the task sensitivity shown by morpho-orthographic effects.

In sum, the present data show that morpho-orthographic segmentation is not always necessary in order to achieve the lexical identification of complex words. By doing so, they challenge in several important ways all existing models of visual word identification. It is possible to see ways in which these models might be modified in order to account for this new evidence; however, it is less clear how these modifications should be specifically implemented computationally, which leaves room for the adjudication process to take place.
Acknowledgments This research was supported by an "FIRBFuturo in Ricerca" grant from the Italian Ministry of Education, University and Research to Davide Crepaldi

Authors' contributions Marco conceived the experiment; Elena and Simona created the stimuli, with contributions from Marco and Davide; Elena and Simona collected the data; Marco and Davide analyzed the data; Marco and Simona drafted the article, which was critically revised by all authors; Davide supervised the project.

\section{References}

Bayen, R.H., Davidson, D. J., \& Bates, D. (2008). Mixed-effects modeling with crossed random effects for subjects and items. Journal of Memory and Language 390-412.

Baayen, R. H., \& Marelli, M. (2010). The directed compound graph of english: An exploration of lexical connectivity and its processing consequences. In Society for the scientific study of reading.

Bertinetto, P. M., Burani, C., Laudanna, A., Marconi, L., Ratti, D., Rolando, C., et al. (2005). Corpus e lessico di frequenza dell'italiano scritto (colfis).

Bueno, S., \& Frenck-Mestre, C. (2008). The activation of semantic memory: Effects of prime exposure, prime-target relationship, and task demands. Memory \& Cognition, 36, 882-898.

Crepaldi, D., Rastle, K., Coltheart, M., \& Nickels, L. (2010). 'Fell' primes 'fall', but does 'bell' prime 'ball'? Masked priming with irregularly-inflected primes. Journal of Memory and Language, 63, 183-99.

Davis, C. J. (2010). The spatial coding model of visual word identification. Psycholgical Review, 117, 713-758.

Diependaele, K., Duñabeitia, J. A., Morris, J., \& Keuleers, E. (2011). Fast morphological effects in first and second language word recognition. Journal of Memory and Language, 64, 344-358.

Duñabeitia, J. A., Kinoshita, S., Carreiras, M., \& Norris, D. (2011). Is morpho-orthographic decomposition purely orthographic? evidence from masked priming in the same-different task. Language \& Cognitive Processes, 26, 509-529.

Feldman, L. B., O’Connor, P. A., \& Moscoso del Prado Martín, F. (2009). Early morphological processing is morphosemantic and not simply morpho-orthographic: A violation of form-thenmeaning accounts of word recognition. Psychonomic Bulletin and Review, 164, 684-691.

Grainger, J., \& Ziegler, J. C. (2011). A dual-route approach to orthographic processing. Frontiers in psychology, 2.

Järvikivi, J., \& Pyykkönen, P. (2011). Sub- and supralexical information in early phases of lexical access. Frontiers in Psychology, 2, 282.

Kuperman, V., Schreuder, R., Bertram, R., \& Baayen, R. H. (2009). Reading of multimorphemic dutch compounds: Towards a multiple route model of lexical processing. Journal of Experimental Psychology. Human Perception and Performance, 35, 876-895.

Longtin, C., Segui, J., \& Hallé, P. (2003). Morphological priming without morphological relationship. Language \& Cognitive Processes, 18, 313-334.

Marelli, M., \& Luzzatti, C. (2012). Frequency effects in the processing of italian nominal compounds: Modulation of headedness and semantic transparency. Journal of Memory and Language, 66 (4), 644-664.

Marslen-Wilson, W. D., Tyler, L. K., Waksler, R., \& Older, L. (1994). Morphology and meaning in the English mental lexicon. Psychological Review, 10, 13-33.

Norris, D. (2006). The bayesian reader: Explaining word recognition as an optimal bayesian decision process. Psychological Review, 113, $327-357$ 
Norris, D., \& Kinoshita, S. (2008). Perception as evidence accumulation and bayesian inference: Insights from masked priming. Journal of Experimental Psychology. General, 137, 434-455.

Rastle, K., \& Davis, M. H. (2008). Morphological decomposition based on the analysis of orthography. Language \& Cognitive Processes, 23, 942-971.

Rastle, K., Davis, M. H., Marslen-Wilson, W. D., \& Tyler, L. K. (2000). Morphological and semantic effects in visual word recognition: A time-course study. Language \& Cognitive Processes, $15,507-537$
Rastle, K., Davis, M. H., \& New, B. (2004). The broth in my brother's brothel: Morpho-orthographic segmentation in visual word recognition. Psychonomic Bulletin \& Review, 11, 10901098.

Rueckl, J. G., \& Aicher, K. A. (2008). Are corner and brother morphologically complex? not in the long term. Language \& Cognitive Processes, 23, 972-1001.

Taft, M. (2004). Morphological decomposition and the reverse base frequency effect. The Quarterly Journal of Experimental Psychology, 57A, 745-765. 